\title{
Maximum Likelihood Estimation of Position in GNSS
}

\author{
Pau Closas, Student Member, IEEE, Carles Fernández-Prades, Member, IEEE, \\ and Juan A. Fernández-Rubio, Senior Member, IEEE.
}

\begin{abstract}
In this letter, we obtain the Maximum Likelihood Estimator of position in the framework of Global Navigation Satellite Systems. This theoretical result is the basis of a completely different approach to the positioning problem, in contrast to the conventional two-steps position estimation, consisting of estimating the synchronization parameters of the in-view satellites and then performing a position estimation with that information. To the authors' knowledge, this is a novel approach which copes with signal fading and it mitigates multipath and jamming interferences. Besides, the concept of Position-based Synchronization is introduced, which states that synchronization parameters can be recovered from a user position estimation. We provide computer simulation results showing the robustness of the proposed approach in fading multipath channels. The Root Mean Square Error performance of the proposed algorithm is compared to those achieved with state-of-the-art synchronization techniques. A Sequential Monte-Carlo based method is used to deal with the multivariate optimization problem resulting from the ML solution in an iterative way.
\end{abstract}

Index Terms-Maximum likelihood estimation, Satellite navigation systems, Position measurement, Synchronization.

\section{INTRODUCTION}

$\mathbf{G}$ LOBAL Navigation Satellite Systems (GNSS) is the general concept used to identify those systems that allow user position computation based on a constellation of satellites. Specific GNSS systems are the well-known american GPS or the forthcoming european Galileo. Both systems rely on the same principle: the user computes its position from measured distances between the receiver and the set of in-view satellites. These distances are calculated estimating the propagation time that transmitted signals take from each satellite to the receiver [1]. Each satellite is uniquely identified by its own directsequence spread-spectrum signal, transmitted synchronously by all satellites. GNSS receivers are only interested in estimating delays of direct path signals, hereafter referred to as line-of-sight-signal (LOSS), as they are the ones that carry information of direct propagation time. Hence, reflections distort the received signal in a way that may cause a bias in delay and carrier-phase estimates [2].

This work has been partially funded by the Spanish/Catalan Science and Technology Commissions and FEDER funds from the European Commission: TEC2006-06481/TCM, TEC2004-04526, TIC2003-05482, 2005SGR-00639, FIT-330503-2006-2 (m:Ciudad) and 2005SGR-00690.

P. Closas and J. A. Fernández-Rubio are with the Dept. of Signal Theory and Communications, Technical University of Catalonia (UPC), Campus Nord D5-117, 08034 Barcelona, Spain (e-mail: \{closas,juan\}@ gps.tsc.upc.edu).

C. Fernández-Prades is with the Centre Tecnològic de Telecomunicacions de Catalunya (CTTC), Parc Mediterrani de la Tecnologia, Av. Canal Olímpic s/n, 08860 Barcelona, Spain (e-mail: carles.fernandez@cttc.cat).
In this letter, we propose a different approach to the positioning problem. Whereas in conventional receivers estimates of Time Difference Of Arrival (TDOA) are needed to geometrically obtain user coordinates, the study herein proposed focuses on the estimation of position directly from received data. Thus, we allow the system to overcome the bias produced by multipath or momentary blockage of satellite links, because in the two-steps approach the estimation of the synchronization parameters is performed independently for each satellite, whereas the proposed direct position estimation is jointly performed taking into account data received from all in-view satellites. The Maximum Likelihood Estimation (MLE) of position is obtained from the MLE of synchronization parameters, regarding the invariance principle of such estimates. The novelty of this approach is that it allows the use of prior information in a natural way thanks to existing motion models, as opposite to synchronization-parameter based positioning approach where the use of prior information is somehow less apparent, as the evolution of these parameters cannot be modeled easily. The aprioristic information regarding user coordinates can either be obtained from existing motion models, delivered by an Inertial Measurement Unit in an ultra-tight integration configuration or by any other possible source of information available concerning the user motion [3].

The letter is organized as follows. In Section II, we expose the signal model considered in digital GNSS receivers, dependent of synchronization parameters (time delay, Doppler shift and carrier phase). In contrast to this approach, we propose a signal model function of all possible variables of the user motion model. Section III addresses the calculation of the MLE and the position-dependent cost function is presented. Computer simulation results are provided in fading multipath channels where conventional Delay Lock Loop (DLL) performance is seriously degraded. Improved versions of the DLL algorithm are employed in GNSS receivers to estimate TDOA, e.g. Narrow [4] and Double Delta [5] Correlator algorithms. Besides, the concept of Position-based Synchronization is presented in section IV, relying on position estimates to obtain an estimate of synchronization parameters. Appendix I contains the proof of the consistency of the proposed estimator.

\section{Signal Model}

Measurements are considered to be a superposition of plane waves corrupted by thermal noise and non-modeled interferences and multipath. The antenna receives $M$ scaled, timedelayed and Doppler-shifted signals corresponding to each in- 
view satellite. The received complex baseband signal is

$$
x(t)=\sum_{i=1}^{M} a_{i} s_{i}\left(t-\tau_{i}\right) \exp \left\{j 2 \pi f_{d_{i}} t\right\}+n(t)
$$

where $s_{i}(t)$ is the transmitted complex baseband low rate BPSK signal spreaded by the pseudorandom code of the $i-$ th satellite, considered known, $a_{i}$ is its complex amplitude, $\tau_{i}$ is the time-delay, $f_{d_{i}}$ the Doppler deviation and $n(t)$ is zeromean additive white Gaussian noise (AWGN) of variance $\sigma_{n}^{2}$.

If a receiver captures $K$ snapshots, the model in equation (1) can be expressed as

$$
\mathbf{x}=\mathbf{a D}(\boldsymbol{v})+\mathbf{n}
$$

where

- $\mathbf{x} \in \mathbb{C}^{1 \times K}$ is the observed signal vector,

- $\mathbf{a} \in \mathbb{C}^{1 \times M}$ is a vector whose elements are the amplitudes of the $M$ received signals $\mathbf{a}=\left[\begin{array}{lll}a_{1} & \ldots & a_{M}\end{array}\right]$,

- $\boldsymbol{v}=\left[\boldsymbol{\tau}^{T}, \mathbf{f}_{d}^{T}\right]^{T} \in \mathbb{R}^{2 M \times 1}$, is a vector containing the time-delay and the Doppler-shift of each satellite,

- $\mathbf{D}(\boldsymbol{v})=\left[\begin{array}{lll}\mathbf{d}\left(t_{0}\right) & \ldots & \mathbf{d}\left(t_{K-1}\right)\end{array}\right] \in \mathbb{C}^{M \times K}$, known as the basis-function matrix, being $\mathbf{d}(t)=\left[\begin{array}{lll}d_{1} & \ldots & d_{M}\end{array}\right]^{T} \in \mathbb{C}^{M \times 1}$, where each component is defined by $d_{i}=s_{i}\left(t-\tau_{i}\right) \exp \left\{j 2 \pi f_{d_{i}} t\right\}$ the delayed-Doppler shifted narrowband signal envelopes,

- $\mathbf{n} \in \mathbb{C}^{1 \times K}$ represents zero-mean AWGN with piecewise constant variance $\sigma_{n}^{2}$ during the observation interval.

\section{A. Pseudorange modeling}

The model exposed in (2) refers to measurements as a function of time-delays, Doppler-shifts and complex amplitudes. Nevertheless, the final objective is to obtain an algorithm to compute position estimates directly from measurements, so that the model must depend on the unknown user position coordinates $\left(\mathbf{p}=[x, y, z]^{T}\right)$ and the bias of the receiver clock $(\delta t)$. Regarding that user position is calculated from timedelay estimates, the non-linear relation between the user's position and the time-delay of the $i$-th satellite is given by the pseudorange $\rho^{i}$ as

$$
\rho^{i}=c \tau^{i}=\varrho^{i}+c\left(\delta t-\delta t^{i}\right)+\epsilon^{i}
$$

where $c$ is the speed of light, satellites are indexed by $i=$ $1, \ldots, M$ and with the following definitions:

- $\tau^{i}$ is the time-delay estimate at the receiver for the signal emitted at the $i$-th satellite.

- $\varrho^{i}=\sqrt{\left(x^{i}-x\right)^{2}+\left(y^{i}-y\right)^{2}+\left(z^{i}-z\right)^{2}}$ is the geometric distance between the receiver and the $i$-th satellite. $\mathbf{p}^{i}=\left[x^{i}, y^{i}, z^{i}\right]^{T}$ are the coordinates of the $i-$ th satellite in the Earth-Centered Earth-Fixed (ECEF) coordinate system, which can be computed from the lowrate navigation message [1].

- $\delta t$ is the bias of the receiver clock w.r.t GPS time, which is unknown.

- $\delta t^{i}$ is the clock bias of satellite $i$ w.r.t. GPS time, known from the navigation message contained in $s_{i}(t)$.

- the term $\epsilon^{i}$ includes errors from various sources such as atmospheric delays, ephemeris mismodeling and relativistic effects among others. In the sequel, it is assumed that these effects can be compensated with differential techniques that are out of the scope of this paper [1].

\section{B. Pseudorange rate modeling}

The observed carrier frequency at the receiver differs from its nominal frequency due to the Doppler effect. These frequency shifts are caused by user-satellite relative motion and by frequency errors and drifts in user and satellite clocks. Accurate Doppler-shift estimates yield to precise velocity calculations, useful in positioning and navigation applications with high user dynamics. The Doppler-shift due to the relative motion of the user and the $i$-th satellite is expressed as

$$
f_{d_{i}}=\left(\frac{\mathbf{v}^{i}-\mathbf{v}}{c} \mathbf{u}^{i}\right) f_{c}
$$

where $\mathbf{v}$ and $\mathbf{v}^{i}$ are the velocity vectors of the user and the $i$-th satellite respectively and $f_{c}$ represents the corresponding carrier frequency used in navigation systems. Being the operator $\|\cdot\|$ the $L^{2}$-norm of a vector, $\mathbf{u}^{i}$ represents the unitary direction vector of the $i$-th satellite relative to the user,

$$
\mathbf{u}^{i}=\frac{\mathbf{p}^{i}-\mathbf{p}}{\left\|\mathbf{p}^{i}-\mathbf{p}\right\|}
$$

Differentiating (3) w.r.t. time, the pseudorange rate $(\dot{\rho})$ regarding the $i$-th satellite is related to the Doppler shift as

$$
\dot{\rho}^{i}=\left(\mathbf{v}^{i}-\mathbf{v}\right) \mathbf{u}^{i}+c(\dot{\delta} t-\dot{\delta} t)+\dot{\epsilon}^{i}=c \frac{f_{d_{i}}}{f_{c}}+c \dot{\delta} t+\epsilon_{f}
$$

being $\dot{\delta} t$ the receiver clock drift and $\epsilon_{f}$ noise on the phase rate measurement and non-modeled terms.

\section{Measurement model as a function of position coordinates}

At this point, we have a relation between the time/frequency parameterization of the model and its corresponding positionbased model, described by equations (3) and (4). Gathering all considered user motion parameters in a real vector $\gamma$, for instance position and velocity $\gamma=\left[\mathbf{p}^{T}, \mathbf{v}^{T}\right]^{T}$, equation (2) can be rewritten to explicitly express its dependence on $\gamma$

$$
\mathbf{x}=\mathbf{a D}(\gamma)+\mathbf{n}
$$

The equivalence between equations (2) and (7) is valid as both time delays and Doppler shifts are injective functions with respect to the motion parameters vector, i.e. given a motion parameter vector, it can only be related to a single pair of time-delay and Doppler-shift vectors.

\section{MaXimum Likelihood Estimation of Position}

We now consider the Maximum Likelihood Estimation (MLE) of signal parameters taking into account the measurement model presented in equation (2), parameterized by time-delays and Doppler-shifts of each satellite. Considering equations (3) and (4), the MLE of receiver position arises thanks to the invariance principle of the ML estimates.

We first take into account that the MLE is equivalent to the solution obtained by a Least Squares (LS) criteria under the assumption of zero-mean AWGN. Neglecting additive and 
multiplicative constants, maximizing the likelihood function of measurement equation (2) is equivalent to minimizing

$$
\Lambda\left(\mathbf{a}, \boldsymbol{\tau}, \mathbf{f}_{d}\right) \triangleq \Lambda(\mathbf{a}, \boldsymbol{v})=\frac{1}{K}\|\mathbf{x}-\mathbf{a D}(\boldsymbol{v})\|^{2}
$$

and with the following cross-correlations estimation definitions

$$
\begin{aligned}
\hat{r}_{x x} & =\frac{1}{K} \mathbf{x x}^{H} & \hat{\mathbf{R}}_{x d}(\boldsymbol{v}) & =\frac{1}{K} \mathbf{x D}^{H}(\boldsymbol{v}) \\
\hat{\mathbf{R}}_{d x}(\boldsymbol{v}) & =\hat{\mathbf{R}}_{x d}^{H}(\boldsymbol{v}) & \hat{\mathbf{R}}_{d d}(\boldsymbol{v}) & =\frac{1}{K} \mathbf{D}(\boldsymbol{v}) \mathbf{D}^{H}(\boldsymbol{v})
\end{aligned}
$$

it is straightforward to obtain the MLE of amplitudes as

$$
\hat{\mathbf{a}}_{M L}=\left.\hat{\mathbf{R}}_{x d}(\boldsymbol{v}) \hat{\mathbf{R}}_{d d}^{-1}(\boldsymbol{v})\right|_{\boldsymbol{\tau}=\hat{\boldsymbol{\tau}}_{M L}, \mathbf{f}_{d}=\hat{\mathbf{f}}_{d_{M L}}}
$$

The ML estimation of synchronization parameters is then obtained by minimizing the nonlinear cost function resulting from the substitution of (10) in (8),

$$
\begin{gathered}
\hat{\boldsymbol{\tau}}_{M L} \quad, \hat{\mathbf{f}}_{d_{M L}}=\underset{\boldsymbol{v}=\left[\boldsymbol{\tau}^{T}, \mathbf{f}_{d}^{T}\right]^{T}}{\arg \min }\{\Lambda(\boldsymbol{v})\} \\
=\arg \min _{\boldsymbol{\tau}, \mathbf{f}_{d}}\left\{\hat{r}_{x x}-\hat{\mathbf{R}}_{x d}\left(\boldsymbol{\tau}, \mathbf{f}_{d}\right) \hat{\mathbf{R}}_{d d}^{-1}\left(\boldsymbol{\tau}, \mathbf{f}_{d}\right) \hat{\mathbf{R}}_{x d}^{H}\left(\boldsymbol{\tau}, \mathbf{f}_{d}\right)\right\}
\end{gathered}
$$

Our aim is to obtain an expression of the likelihood function dependent on $\gamma$, that is as a function of user position instead of the synchronization parameters. Notice that $\tau \triangleq \tau(\gamma)$ and $f_{d} \triangleq f_{d}(\gamma)$, as described by equations (3) and (4). Thus the MLE of user position is given by the vector $\gamma$ that maximizes the likelihood function or, equivalently, the vector $\gamma$ that minimizes $\Lambda(\gamma)$, thanks to the invariance principle of the ML estimates under injective functions [6]. Hence,

$$
\begin{aligned}
\hat{\gamma}_{M L} & =\arg \min _{\gamma}\{\Lambda(\gamma)\} \\
& =\arg \min _{\gamma}\left\{\hat{r}_{x x}-\hat{\mathbf{R}}_{x d}(\gamma) \hat{\mathbf{R}}_{d d}^{-1}(\gamma) \hat{\mathbf{R}}_{x d}^{H}(\gamma)\right\}
\end{aligned}
$$

Whereas in the synchronization-parameter based positioning a two-dimensional optimization has to be performed for each tracked satellite, the position-dependent cost function takes into account signals coming from all satellites to obtain a position estimate, dealing with a single multivariate optimization problem for all the received satellites. For the sake of clarity and without loss of generality, we now consider that one of the coordinates (say $z$ ) and the receiver clock bias are known (or vary slowly with time and can be tracked by other methods) so that we can plot the three-dimensional likelihood function. Figure 1 shows the cost function in equation (12) in a realistic scenario composed of 7 satellites evaluated for different coordinate errors, denoted as $\epsilon_{x}$ and $\epsilon_{y}$. Gradientlike methods can be used to iteratively minimize the cost function such as the Newton-Raphson algorithm. However, these methods highly depend on a proper initialization to converge to the optimal value due to the high non-linearity of the function. Alternative methods must be studied to deal with the optimization in a more suitable and implementable way. To this aim, Sequential Monte-Carlo (SMC) methods, a set of statistical simulation-based methods [7], have been investigated and adapted to the multivariate optimization problem at hand [8]. Basically, the algorithm generates a set of support points in which the ML cost function is evaluated, the trial

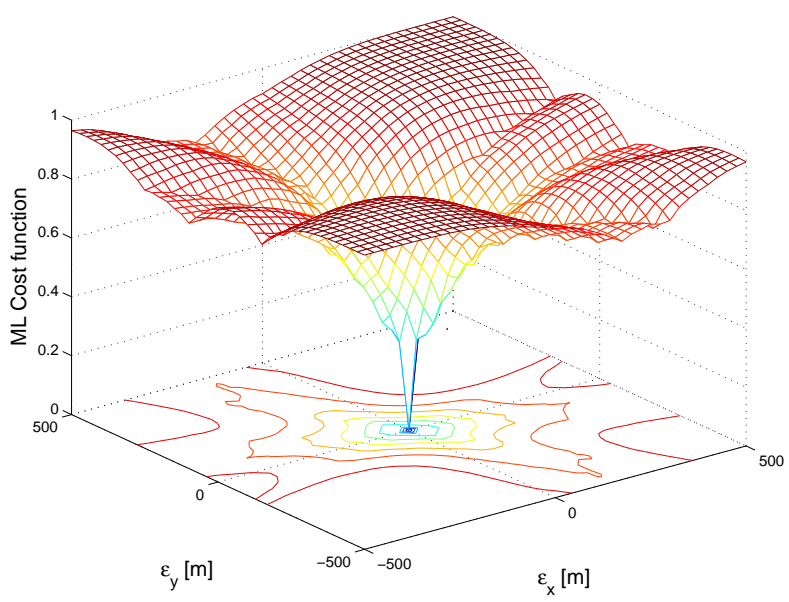

Fig. 1. The ML cost function in equation (12) as a function of the unknown 2-D user position, $\gamma=[x, y]^{T}$.

point associated to the lowest weight is then propagated to the next iteration until convergence.

In some applications, it might be desirable to provide external information to the system regarding receiver motion, aiming at improving performance. SMC methods provide an appealing way to introduce prior information in the estimation algorithm. Hence, the possibility of using aprioristic information can easily be taken into account when optimizing the ML with the SMC method used herein. Prior information can improve, not only the accuracy of the estimates but the convergence time allowing the system to deal with more aggressive channel characteristics than a conventional ML approach does. However, the use of prior information is out of the scope of this letter and is a key issue for future work.

\section{THE CONCEPT OF POSITION-BASED SYNCHRONIZATION}

Although estimates obtained with the proposed approach are the user coordinates themselves, it might be desirable to obtain synchronization parameters. This can be accomplished by undoing the transformations in (3) and (6), being injective functions. The estimation of synchronization parameters relying on position estimates is hereafter referred to as Positionbased Synchronization, used as a figure of merit. We now consider a multipath replica in the scenario, with a signal-tomultipath ratio of $3 \mathrm{~dB}$. In Figures 2 and 3, the performance of both the MLE of position with SMC optimization and conventional DLL-based single-point approach [1] are compared in terms of positioning error, evidencing great improvements in the ML approach. Multipath envelopes obtained outperform those of DLL algorithms, having direct impact in pseudorange estimation and in position accuracy. This is a useful approach when tracking satellites with low carrier-to-noise density ratios, for instance, in indoor navigation or in environments where the loss of tracking with certain satellites might occur due to severe fading conditions and signal blockages, among other scenario-dependant nuisance effects. Considering that position is jointly estimated regarding information of all inview satellites, a diversity is introduced in this process as the 


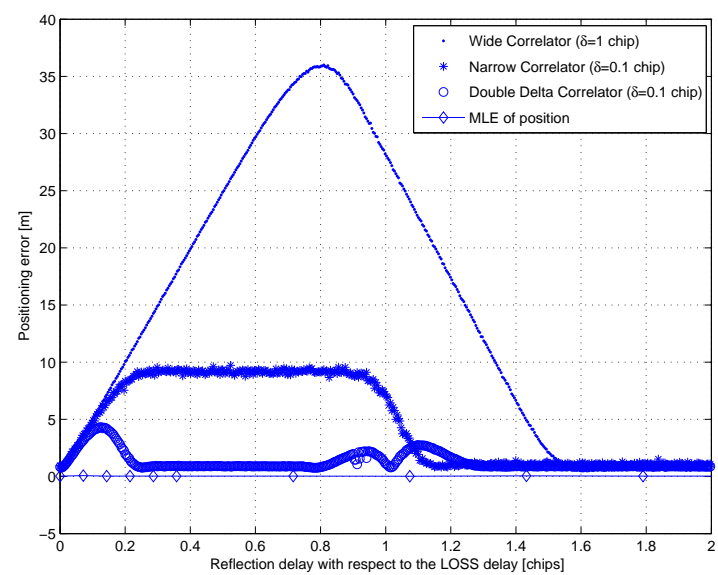

Fig. 2. RMSE of position $(\|\mathbf{p}-\hat{\mathbf{p}}\|)$ under multipath conditions.

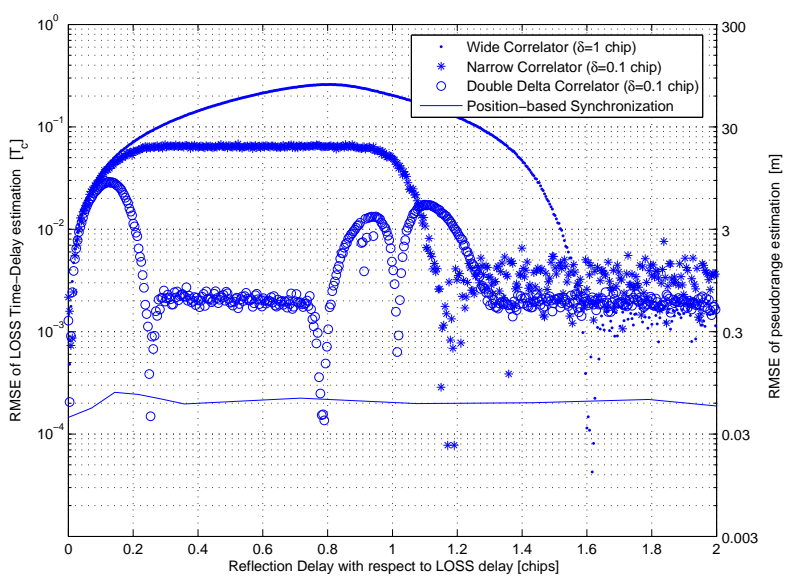

Fig. 3. Time-delay RMSE along with its impact in pseudorange estimation of this satellite.

propagation path for each satellite link is different. Positionbased Synchronization takes advantage of this diversity.

\section{CONCLUSIONS}

In this letter, the MLE of position coordinates has been obtained for GNSS taking into account the invariance principle of the ML estimates. This theoretical result is the basis of a novel approach to the positioning problem. Conventional receivers estimate synchronization parameters of the in-view satellites and then perform a position estimation with that information. In our approach, the problem is reduced to a single multivariate optimization problem targeting user position and, optionally, other motion parameters. This approach is robust against signal fading and mitigates multipath and jamming interferences as the estimation of position is jointly performed taking into account measurements from all in-view satellites. Thus, if a satellite link is severely degraded by channel characteristics, the rest of the in-view constellation can overcome the nuisance in the global estimate. The main drawback of this approach is the lack of a computationally efficient optimization algorithm, due to the high dimensionality of the problem. SMC methods are serious candidates for the optimization step, providing an appealing and natural way of introducing prior information in the motion estimation process. In addition, we have introduced the concept of Position-based Synchronization, showing that synchronization parameters can be recovered from position estimates, with better accuracy results than conventional synchronization algorithms.

\section{APPENDIX I \\ CONSISTENCY OF THE MAXIMUM LiKELIHOOD ESTIMATOR OF POSITION}

An estimator of a parameter $\gamma$ is said to be consistent if its estimates converge in probability to the true value $(\tilde{\gamma})$ of the parameter as $K \rightarrow \infty$. The asymptotic values of the correlation terms in (9) are

$$
\begin{aligned}
\lim _{K \rightarrow \infty} \hat{\mathbf{R}}_{x d} & =\lim _{K \rightarrow \infty} \frac{1}{K}(\mathbf{a D}(\tilde{\gamma})+\mathbf{n}) \mathbf{D}^{H}(\boldsymbol{\gamma})=\mathbf{a} \mathbf{C}_{d d}(\tilde{\gamma}, \boldsymbol{\gamma}) \\
\lim _{K \rightarrow \infty} \hat{r}_{x x} & =r_{x x}, \quad \lim _{K \rightarrow \infty} \hat{\mathbf{R}}_{d d}=\mathbf{C}_{d d}(\boldsymbol{\gamma}, \boldsymbol{\gamma}) \\
\text { where } & \mathbf{C}_{d d}(n, m)=\lim _{K \rightarrow \infty} \frac{1}{K} \mathbf{D}(n) \mathbf{D}^{H}(m)
\end{aligned}
$$

The limit of the ML cost function is constructed from substitution of the latter expressions in $\Lambda(\gamma)$, then we have added and subtracted $\mathbf{a C}_{d d}(\tilde{\gamma}, \tilde{\gamma}) \mathbf{a}^{H}$

$$
\begin{aligned}
\lim _{K \rightarrow \infty} \Lambda(\boldsymbol{\gamma}) & =r_{x x}-\mathbf{a} \mathbf{C}_{d d}(\tilde{\boldsymbol{\gamma}}, \boldsymbol{\gamma}) \mathbf{C}_{d d}^{-1}(\boldsymbol{\gamma}, \boldsymbol{\gamma}) \mathbf{C}_{d d}^{H}(\tilde{\boldsymbol{\gamma}}, \boldsymbol{\gamma}) \mathbf{a}^{H} \\
& =\mathbf{a} \boldsymbol{\Omega} \mathbf{a}^{H}+\lim _{K \rightarrow \infty} \Lambda(\tilde{\gamma}) \\
\boldsymbol{\Omega} & =\mathbf{C}_{d d}(\tilde{\boldsymbol{\gamma}}, \tilde{\gamma})-\mathbf{C}_{d d}(\tilde{\boldsymbol{\gamma}}, \boldsymbol{\gamma}) \mathbf{C}_{d d}^{-1}(\boldsymbol{\gamma}, \boldsymbol{\gamma}) \mathbf{C}_{d d}^{H}(\tilde{\boldsymbol{\gamma}}, \boldsymbol{\gamma})
\end{aligned}
$$

now we have to proof that $\tilde{\gamma}$ minimizes the ML cost function

$$
\lim _{K \rightarrow \infty} \Lambda(\gamma) \geq \lim _{K \rightarrow \infty} \Lambda(\tilde{\gamma}) \quad, \forall \gamma
$$

which occurs if $\Omega$ is a non-negative definite matrix, since is straightforward to prove that $\Lambda(\gamma)$ is a positive definite matrix. Notice that $\boldsymbol{\Omega}$ is the Schur complement of $\mathbf{C}_{d d}(\gamma, \gamma)$ in the matrix formed as

$$
\left(\begin{array}{ll}
\mathbf{C}_{d d}(\tilde{\gamma}, \tilde{\gamma}) & \mathbf{C}_{d d}(\tilde{\gamma}, \boldsymbol{\gamma}) \\
\mathbf{C}_{d d}^{H}(\tilde{\boldsymbol{\gamma}}, \boldsymbol{\gamma}) & \mathbf{C}_{d d}(\boldsymbol{\gamma}, \boldsymbol{\gamma})
\end{array}\right)=\lim _{K \rightarrow \infty} \frac{1}{K}\left(\begin{array}{c}
\mathbf{D}(\tilde{\gamma}) \\
\mathbf{D}(\boldsymbol{\gamma})
\end{array}\right)\left(\begin{array}{l}
\mathbf{D}^{H}(\tilde{\gamma}) \\
\mathbf{D}^{H}(\boldsymbol{\gamma})
\end{array}\right)^{T}
$$

being the matrix non-negative, due to its quadratic form, any Schur complement of it is also non-negative, q.e.d.

\section{REFERENCES}

[1] B. Parkinson and J. Spilker, Eds., Global Positioning System: Theory and Applications, vol. I, II, ser. Progress in Astronautics and Aeronautics. Washington DC: American Institute of Aeronautics, Inc., 1996.

[2] R. D. J. V. Nee, "Spread-Spectrum Code and Carrier Synchronization Errors Caused by Multipath and Interference," IEEE Trans. Aerosp. Electron. Syst., vol. 29, no. 4, pp. 1359-1365, October 1993.

[3] M. S. Grewal, L. R. Weill, and A. P. Andrews, Global Positioning Systems, Inertial Navigation, and Integration. John Wiley \& Sons, 2001.

[4] A. J. V. Dierendonck, P. Fenton, and T. Ford, "Theory and Performance of Narrow Correlator Spacing in a GPS Receiver," Navigation: Journal of The Institute of Navigation, vol. 39, no. 3, pp. 265-283, Fall 1992.

[5] G. McGraw and M. Braash, "GNSS Multipath Mitigation Using Gated and High Resolution Correlator Concepts," in Proceedings of the ION GPS/GNSS 1999, 1999, pp. 333-342.

[6] A. Papoulis and S. U. Pillai, Probability, Random Variables and Stochastic Processes, 4th ed. McGraw-Hill, 2001.

[7] A. Doucet, N. de Freitas, and N. Gordon, Eds., Sequential Monte Carlo Methods in Practice. Springer, 2001.

[8] P. Closas, C. Fernández-Prades, and J. A. Fernández-Rubio, "Optimizing the Likelihood with Sequential Monte-Carlo methods," in XXI National Symposium of the International Union of Radio Science (URSI), Oviedo, Spain, 2006. 